\title{
THE DIFFRACTIVE LOGARITHMIC SLOPE AND THE SATURATION PHENOMENA
}

\author{
M.B. GAY DUCATI ${ }^{\star}$, V.P. GONÇALVES ${ }^{\dagger}$, M.V.T. MACHADO* \\ * Instituto de Física, Universidade Federal do Rio Grande do Sul \\ Caixa Postal 15051, 91501-970 Porto Alegre, RS, BRAZIL. \\ E-mail: gay@if.ufrgs.br,magnus@if.ufrgs.br \\ † Instituto de Física e Matemática, Universidade Federal de Pelotas \\ Caixa Postal 354, CEP 96010-090, Pelotas, RS, BRAZIL \\ E-mail:barros@ufpel.tche.br
}

\begin{abstract}
The logarithmic slope of the diffractive structure function is a potential observable scanning the hard and soft contributions in diffraction, allowing to disentangle the QCD dynamics. We report our calculations concerning this quantity, in particular the estimates emerging from the saturation model applied to diffraction dissociation.
\end{abstract}

\section{Introduction}

The measurements of the derivative quantity $F_{2}$-slopel on $Q^{2}$ allowed a renewed interest of testing the matching of hard and soft approaches and provided constraints for the saturation formalisms. The reported turnover on the $x$ dependence was readly associated with the transition region between the interplaying domains. The most refent determinations of the slope still deserve a better theoretical description $\mathrm{B}$, keeping a turnover pattern at fixed c.m.s. energy $W$. When we focus in diffractive DIS, in particular the structure function $F_{2}^{D}$, the situation is far from clear: initially considered as a predominantly soft process, the experimental results suggests that the diffractive cross section at HERA contains hard and soft components. However, the hard piece is stronger than the previously expected. Fortunately, if we consider the pQCD approach, diffraction stands a more profitable field to study saturation effects than the inclusive case. This comes from the fact that in DDIS the interaction probes larger dipole size configuration (soft content) than in the DIS reactionl. Although the quite different pictures considered to interpret the diffractive measurements, almost all of them fit the data set properly 6 . We have proposed a derivative quantity, the diffractive logarithmic slope, which would help to disentangle the underlying dynamics in diffractive DIS, settling its validity range, if such observable is measured. In Ref.l one perform studies of the proposed quantity for two sound models, representing the essential features in both Regge and pQCD formalisms. It was found that 
important deviations in the predictions between the models emerge, considering the available kinematic spectrum in diffractive DIS $\mathbf{6}$. Here we report in particular the calculations considering the saturation pQCD model applied to diffraction dissociation and its comparison with the non-satured QCD approach, performed in details in $\mathrm{G}$. Such results allow to verify the role played by the saturation effects in these processes and the extension of the standard QCD formalism to kinematical regions forbidden perturbatively.

\section{The Diffractive Logarithmic Slope and the Saturation Phenomenon}

In perturbative QCD, the $\gamma^{*} p$ process is described in terms of the photon splitting into a $q \bar{q}$ pair, far upstream of the nucleon, which then scatters in the proton. This reaction is mediated by the one gluon exchange which turns out into a multi-gluon one when the saturation region is approached. A remarkable feature is that the mechanism leading to the photon dissociation and the further scattering has a factorizable form, written in terms of a convolution between a photon wavefunction (dipole transverse size $r$ and quark longitudinal momentum fraction $z$ ) and a $q \bar{q}$ cross section. For transverse and longitudinally polarized photons the $\gamma^{*} p$ cross section has the form

$$
\sigma_{T, L}\left(x, Q^{2}\right)=\int d^{2} \mathbf{r} \int_{0}^{1} d \alpha\left|\Psi_{T, L}(\alpha, \mathbf{r})\right|^{2} \hat{\sigma}\left(x, r^{2}\right),
$$

where $\Psi_{T, L}$ are the photon wavefunctions. Although they are calculated from perturbation theory, the $q \bar{q}$ cross section, $\hat{\sigma}$, has strong nonperturbative contributions that should be modeled. We choose the saturation modele, which reproduces the experimental results at both inclusive and diffractive electroproduction. The dynamics of saturation is present in the effective dipole cross section in an eikonal way $\hat{\sigma}\left(x, r^{2}\right)=\sigma_{0}\left[1-\exp \left(-\frac{r^{2}}{4 R_{0}^{2}(x)}\right)\right]$, where the $x$ dependent saturation scale is $R_{0}(x)=\frac{1}{Q_{0}}\left(\frac{x}{x_{0}}\right)^{\lambda / 2}$. The normalization $\sigma_{0}$ and the remaining parameters were determined from data with $x<10^{-2}$. This model consistently interpolates between the saturation regime and the scaling regime of $\sigma_{T}\left(F_{2}\right)$. The elastic scattering of the $q \bar{q}$ pair dominates the diffractive $\gamma^{*} p$ process for not too large values of the diffractive mass $M_{X}$. Instead, at large $M_{X}$ the emission of a gluon becomes the leading contribution. Then, the diffractive cross section is, given the slope $B_{D}$,

$$
\sigma^{D}\left(x, Q^{2}\right)=\frac{1}{B_{D}} \frac{1}{16 \pi} \int d^{2} \mathbf{r} \int_{0}^{1} d \alpha\left|\Psi_{T, L}(\alpha, \mathbf{r})\right|^{2} \hat{\sigma}^{2}\left(x, r^{2}\right) .
$$



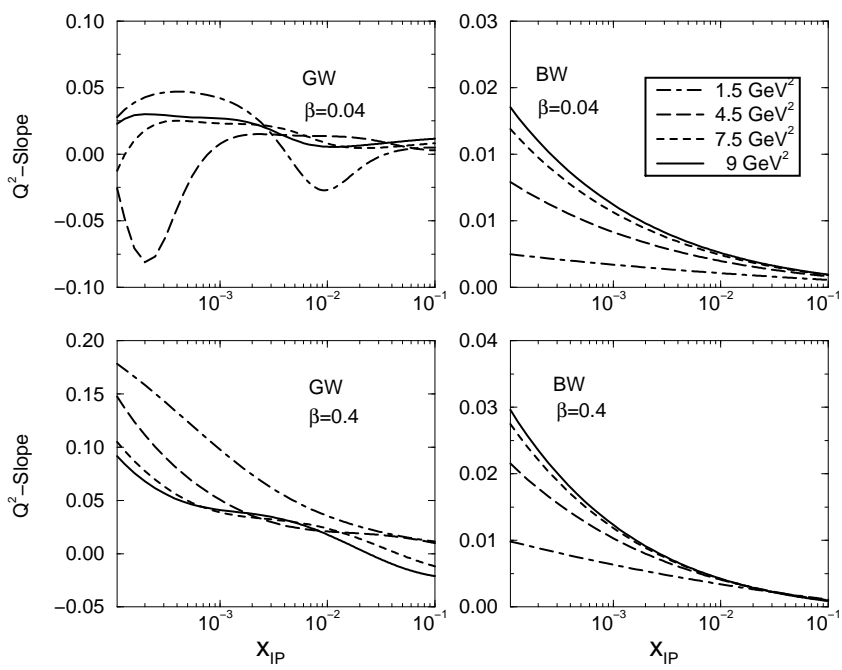

Figure 1. The $x_{\mathbb{P}}$ dependence on the logarithmic slope for the pQCD model (BW) and the the saturation model (GW) presented at typical $\beta$ values.

In ref. 5 it has been found a ratio between the diffractive and the inclusive cross sections roughly constant, and that the diffractive cross section is sensitive to the infrared cutoff which is effectively provided by $2 R_{0}(x)$ concluding that diffraction probes the transition region. We performed numerically the $F_{2}^{D}$ logarithmic slope, using the same parameters values from Ref. 3 . In Fig. (11), one shows the $x_{\mathbb{P}}$ behavior for both the approaches with 5 and without saturation 10 , at typical $\beta$ values. We analyze in particular the transition region between hard and soft dynamics, settled by the low virtualities $Q^{2} \sim 1.5-9 \mathrm{GeV}^{2}$. The saturation model produces a transition between positive and negative slope values at low $\beta=0.04$, while presents a positive slope for medium and large $\beta$. This feature is corroborated by the preliminary ZEUS studies 11 . The pQCD approach without saturation 10 , shows a positive slope for the whole $Q^{2}$ and $x_{\mathbb{P}}$ range. In 9 , one observed such a smooth behavior for the $\mathrm{pQCD}$ predictions, with a positive slope in almost the whole range, whereas a sound Regge model predicted predominantly negative slope values in this kinematical domain, converging to a flat value for larger $x_{\mathbb{P}}$. A clear difference between the predictions of Regge and QCD is the change of signal of the slope with the $Q^{2}$ evolution at medium $\beta$. Returning, since the diffractive cross section is strongly sensitive to the infrared cutoff, one of the main differences between these models is the assumption related to the 
small $Q^{2}$ region. In the pQCD models without saturation, an ad hoc cutoff in the transverse momentum is inserted, as well as the energy dependence of the unintegrated gluon distribution. In the saturation model, instead, the saturation radius $R_{0}(x)$ gives the infrared cutoff (the saturation momentum scale) and determines the energy dependence. If this scale is large $\left(1-2 \mathrm{GeV}^{2}\right)$, then the resulting process is not soft and can be completely calculated using pQCD methods. Therefore, the saturation model extends the pQCD approach towards lower $Q^{2}$ values. We conclude that, the difference between the behaviors predicted by these two models for the $x_{\mathbb{P}}$ spectrum, mainly in the region of small $\beta$ and medium $Q^{2}$, is large, which should allow to discriminate the dynamics in future experimental analyzes.

\section{References}

1. A. Caldwell, Invited talk, DESY Theory Workshop. DESY, Hamburg (Germany) October 1997. J. Breitweg et al., Eur. Phys J. C7, 609 (1999).

2. H1 Collaboration, Eur. Phys. J. C21, 33 (2001); ZEUS Collaboration. A study of scaling violations in the proton structure function $F_{2}$, in Proc. Conference ICHEP2000, Osaka, Japan (2000).

3. R. Thorne, in Proc. Workshop on Low x Physics 2001, June 27 - 30, Krakow, Poland (2001).

4. H1 Coll. Z. Phys. C76, 613 (1997); ZEUS Coll. Eur. Phys. J. C1, 81 (1998).

5. K. Golec-Biernat, M. Wüsthoff, Phys. Rev. D59, 014017 (1999); Phys. Rev. D60, 114023 (1999).

6. H1 Collaboration, Measurement of the Diffractive Structure Function $F_{2}^{D(3)}\left(x_{\mathbb{P}}, \beta, Q^{2}\right)$ at HERA, in Proc. Conference EPS2001, Budapest, July 12 (2001); in Proc. Conference Lepton-Photon2001, July 23 (2001).

7. M.B. Gay Ducati, V.P. Gonçalves, M.V.T. Machado, Phys. Lett. B506, 52 (2001).

8. M.B. Gay Ducati, V.P. Gonçalves, M.V.T. Machado, in Proc. IXth Blois Workshop, Pruhonice near Prage, June 9-15 (2001) hep-ph/0110025.

9. M.B. Gay Ducati, V.P. Gonçalves, M.V.T. Machado, Nucl. Phys. A (in press) hep-ph/0103245.

10. J. Bartels, M. Wüsthoff, J. Phys. G: Nucl. Part. Phys. 22, 929 (1996); J. Bartels, C. Royon, Mod. Phys. Lett. A14, 1583 (1999).

11. ZEUS Collaboration, Eur. Phys. J. C6, 67 (1999). ZEUS Collaboration, Measurement of the diffractive cross section at $Q^{2}<1 G e V^{2}$ at HERA, in Proc. Conf. ICHEP2000, Osaka, Japan (2000).

ggmismd2001: submitted to World Scientific on November 13, 2018 